\title{
Distribution of localized states from fine analysis of electron spin resonance spectra of organic semiconductors: Physical meaning and methodology
}

\author{
Andrey S. Mishchenko ${ }^{1,2}$, Hiroyuki Matsui ${ }^{3}$, and Tatsuo Hasegawa ${ }^{3}$ \\ ${ }^{1}$ Cross-Correlated Materials Research Group (CMRG), RIKEN Advanced Science Institute (ASI), Wako 351-0198, Japan \\ ${ }^{2} R R C$ "Kurchatov Institute" - 123182 - Moscow - Russia \\ ${ }^{3}$ National Institute of Advanced Industrial Science and Technology (AIST), AIST Tsukuba Central 4, Tsukuba 305-8562, Japan
}

(Dated: March 24, 2021)

\begin{abstract}
We develop an analytical method for the processing of electron spin resonance (ESR) spectra. The goal is to obtain the distributions of trapped carriers over both their degree of localization and their binding energy in semiconductor crystals or films composed of regularly aligned organic molecules [Phys. Rev. Lett. 104, 056602 (2010)]. Our method has two steps. We first carry out a fine analysis of the shape of the ESR spectra due to the trapped carriers; this reveals the distribution of the trap density of the states over the degree of localization. This analysis is based on the reasonable assumption that the linewidth of the trapped carriers is predetermined by their degree of localization because of the hyperfine mechanism. We then transform the distribution over the degree of localization into a distribution over the binding energies. The transformation uses the relationships between the binding energies and the localization parameters of the trapped carriers. The particular relation for the system under study is obtained by the Holstein model for trapped polarons using a diagrammatic Monte Carlo analysis. We illustrate the application of the method to pentacene organic thin-film transistors.
\end{abstract}

PACS numbers: 85.30.Tv, 73.20.At, 73.61.Ph, 76.30.-v

\section{INTRODUCTION}

The electron spin resonance (ESR) technique offers a unique microscopic probe of carriers in semiconductors with unpaired spins. It measures transitions between the quantum levels $m_{s}= \pm 1 / 2$ in the presence of a magnetic field $\underline{1-4}$. The spectrum of the transition between two quantum levels constitutes a $\delta$-function provided there is no external interference with the quantum levels of the spin system. However, the quantum states of carrier spins undergo a variety of interactions with the environment. This interaction destroys the $\delta$-functional form of the spectrum and broadens it. The broadening of the ESR signal is a result of two fundamentally different contributions from the medium. The first is a decay of the quantum levels caused by interaction with the excitations of the environment. This mechanism leads to the Lorentzian shape of the spectral line. The second contribution is a result of the inhomogeneities of the medium. One example is the interaction with nuclear spins that is known as hyperfine interaction; here the inhomogeneities are caused by the probability distribution of the nuclear spin moments. In this case the spectroscopic signal is the sum of the contributions from spin systems located in different surroundings. The energy of signal in every surrounding is shifted by the local magnetic field depending on the environment so that the summed signal has an inhomogeneous shape.

As an example, electronic spin of cationic pentacene molecule isolated in a solution exhibits inhomogeneous broadening of the ESR signal that arises because of hyperfine coupling with 14 proton nuclear spins. The signal is constituted of a series of individual lines due to the hyperfine splitting. The envelope function of the signal is roughly reproduced by a Gaussian ${ }^{5-7}$. This feature is consequence of the central limit theorem (CLT). The local magnetic field for each electronic spin is caused by interaction with 14 proton nuclear spins and the random energy shifts of respective ESR signals are inevitably spread in accordance with Gaussian distributions, as a result of the independent nature of respective nuclear spin orientations.

We note that the anisotropic values of $g$-factors are averaged out (or narrowed motionally) by the rotation motion of the molecules in solution. In contrast, solid-state organic molecular crystals exhibit ESR spectra composed of individual lines that are broadened due to the faster decay rate. Then, there might be the case that the individual lines have to become unresolved and the resulting ESR spectrum has to be very close to Gaussian envelope.

Recent major developments in the field of organic thinfilm transistors (TFTs) allow high-precision field-induced ESR measurement (which is referred in the following simply as ESR) for the carriers in semiconductor crystals or films composed of regularly aligned organic molecules. In the measurements, carries are doped without introducing any randomness by using the field-induced technique. The ESR signal in organic TFTs was first measured and analyzed in the groundbreaking study by Marumoto and his coauthors ${ }^{8.9}$. The ESR signal observed in pentacene TFT at room temperature appeared to be narrower than that observed in solution. The authors claimed that the narrower linewidth should be an evidence of a spatial extension of wavefunction. According to CLT, the linewidth of a signal coming from charge distribution covering $N$ molecules is narrower by the factor $1 / \sqrt{N}$. Then, 
assuming that the signal is Gaussian it was concluded that the wavefunction is spread over $N \approx 10$ molecules.

However, we note that the analyses were performed on the ESR spectrum with non-Gaussian lineshape. Subsequent studies have shown that the field-induced ESR signal and linewidth is temperature dependent $\underline{\underline{10}} \underline{-12}$. Typical pentacene TFTs and rubrene single-crystal transistors exhibit sharp ESR signal whose single-Lorentzian linewidth presents motional narrowing ${ }^{13}$ effects with increase of the temperature. In case of the pentacene TFTs, the feature is well consistent with the thermally activated multiple trap-and-release (MTR) transport with the activation energy of about $10 \mathrm{meV}$ in the high temperature range. In contrast, the narrowing effect by the increase of temperature is not observed below around $50 \mathrm{~K}$. Actually it was demonstrated by the continuous wave saturation experiments that all carriers in the pentacene TFTs are localized at $T<50 \mathrm{~K}$ and all relaxation channels are frozen at such low temperatures $\underline{14}$. However the ESR spectrum is still deviated from the simple Gaussian at low enough temperature. Therefore, the deviation of the ESR signal from Gaussian shape is not a result of relaxation or motional narrowing but should be associated with the nature of weakly-localized carrier states in the organic TFTs. It is of current important theoretical and experimental challenges in materials physics to understand the carrier transport of organic electronics devices, as they permit productions of large-area and flexible electronic products 15,16 .

In the present paper we analyze the situation when the ESR signal of a semiconducting organic molecular system is a smooth curve that deviates from the Gaussian linewidth even at very low temperatures where the carriers are localized. Here we assume that peculiar lineshape is caused by a further specific inhomogeneity of the pentacene TFTs, as associated with the distribution of weakly-localized carrier states which are responsible for the device operation. Given this assumption, we have developed a unique technique for obtaining the trap density of states from a few to tens of meVs in pentacene TFTs 14 the algorithm of which is described in detail in this paper. Note that the obtained energy resolution for the trap density of states is much higher than that by other methods based on transport or optical measurement $\underline{17}-23$. The mathematical method suggested here is rather general and can be applied to analyses of broad spectrum in a variety of problems outside of those considered here.

In Section II we study the deviation of the inhomogeneous low-temperature ESR signal from the Gaussian shape. We show that the signal from the one pentacene molecule is very similar to a Gaussian showing almost no individual lines from hyperfine splittings. It appears that the ESR lineshapes of a signal from many independent traps of the same kind must be a Gaussian (see IA IIB) whose width is uniquely determined by a single localization parameter $N_{\text {eff }}$, namely an effective number of sites where carrier is localized. Therefore, it is concluded that the non-Gaussian lineshape can be ascribed to the super- position of signals from different kinds of traps, where each kind of trap is described by its own localization parameters $N_{\text {eff }}$. In Section [IC we derive an explicit relationship between the shape of the experimental ESR signal and the distribution of the traps over the localization parameters $N_{\text {eff }}$. This relationship is a Fredholm integral equation of the first kind where unknown function is a distribution of traps. Section III presents an algorithm to solve it based on the stochastic optimization method $(\mathrm{SOM})^{24-27}$. We describe the SOM and present an analysis of its sensitivity to the experimental noise in Sections [IA and IIB respectively. Sections II C.IIID present experimental details, handling realistic noisy experimental data by SOM, and results for traps distributions in pentacene TFTs. Section IIIE presents methods that find the limits of the reliability of the distributions obtained.

Section IV shows how the distribution of the traps over the localization parameter $N_{\text {eff }}$ can be mapped as a distribution over the binding energies $E_{B}$. This transformation can be formulated generally although a particular implementation of the mapping requires the explicit $E_{B}-N_{\text {eff }}$ relationship between the binding energy $E_{B}$ and the localization parameter $N_{\text {eff }}$. This relationship for a given model can be obtained using the exact numeric diagrammatic Monte Carlo method ${ }^{28}$, the analytic momentum average method ${ }^{29.30}$, the coherent basis states $\operatorname{method}^{31.32}$, or numerous other methods (see [33] for a review). We consider in Section IVA the model of two dimensional Holstein polaron in the field of an onsite attractive center. The distribution of the trapped states over the binding energies $E_{B}$ in pentacene TFTs are shown in Section IVB Sections $\nabla$ presents a discussion of our results and Section VI provides conclusive remarks.

\section{ESR SPECTRA OF TRAPPED CARRIERS IN ORGANIC SEMICONDUCTORS: FUNDAMENTAL KNOWLEDGE AND FURTHER GENERALIZATIONS}

In this section, we introduce the well-known characteristic features of the ESR spectra of a single molecule and a cluster containing several molecules (II A $)$. We then present an analysis of a noticeably different case where the carrier is localized on a single impurity in a crystal (IIB). Finally, we consider the case of traps of different origin where we can introduce a relationship between the lineshape of an experimental ESR signal and the distribution of the impurities over different localization parameters (IIC). 


\section{A. ESR spectra for single molecule and a cluster containing several molecules}

In this section, we consider a molecular crystal in which single molecule contains so many nuclear spins that its ESR spectrum has an inhomogeneous Gaussian shape. A typical situation for a carrier trapped in a molecular crystal is that it is localized in a trap and its distribution over the molecular crystal sites $i$ is characterized by a probability distribution $\left\{p_{i}\right\}$. The temperature is assumed to be sufficiently low that we can neglect the "homogeneous" relaxation leading to the Lorentzian shape of the ESR signal. It is also low enough to avoid selfaveraging of the inhomogeneities by the "motional narrowing" mechanism.

In this case the lineshape of the ESR signal is determined by the "inhomogeneous" broadening caused by the site dependent distribution of the hyperfine interactions. When the typical width of the individual spectral lines of the split with hyperfine interaction quantum levels is larger than the typical energy distance between these levels ${ }^{3,6}$, the lineshape of the ESR signal is Gaussian. This shape occurs when the carrier is localized in either a single molecule or a cluster containing several molecules.

The case of a carrier trapped in a crystal is noticeably different from the case of a cluster with several molecules. The probability distribution over $N$ molecules $i\left\{p_{i}, i=1, N\right\}$ is uniform $p_{i}=1 / N$ in a cluster. In contrast, the probability distribution in a crystal trap $p_{i}$, which is density of the carrier in given site $i$, is not uniform, and the only restriction is the normalization condition $\sum_{i} p_{i}=1$. However, as shown below, the ESR signal of a carrier in a trap always retains the Gaussian shape and the width is uniquely determined by the carrier probability distribution $\left\{p_{i}\right\}$.

The simplest ESR signal considered in our study is that for a single molecule. The fine structure of the ESR absorption by a single molecule in a condensed environment is frequently blurred by the broadening of the hyperfine levels. The ESR signal from a single molecule in this case is Gaussian. The standard expression describing the hyperfine structure of one molecule is ${ }^{6}$

$$
\begin{aligned}
R(B)= & \sum_{m_{1}=-n_{1} I_{1}}^{n_{1} I_{1}} \ldots \sum_{m_{k}=-n_{k} I_{k}}^{n_{k} I_{k}} P\left(m_{1}, \ldots, m_{k}\right) \times \\
& \frac{1}{\pi} \frac{\Gamma}{\left(B-\sum_{i=1}^{k} A_{i} m_{i}\right)^{2}+\Gamma^{2}}
\end{aligned}
$$

Here $k$ is the number of groups of equivalent nuclei, $n_{i}$ is the number of equivalent nuclei in the $i$ th group, $I_{i}$ is the nuclear spin in the $i$ th group, $\Gamma$ is the linewidth of each peak, $\mathrm{P}$ is the intensity of each peak and $B$ is the magnetic field. If protons $(I=1 / 2)$ are the only paramagnetic nuclei, as is the case for pentacene molecules, $P$

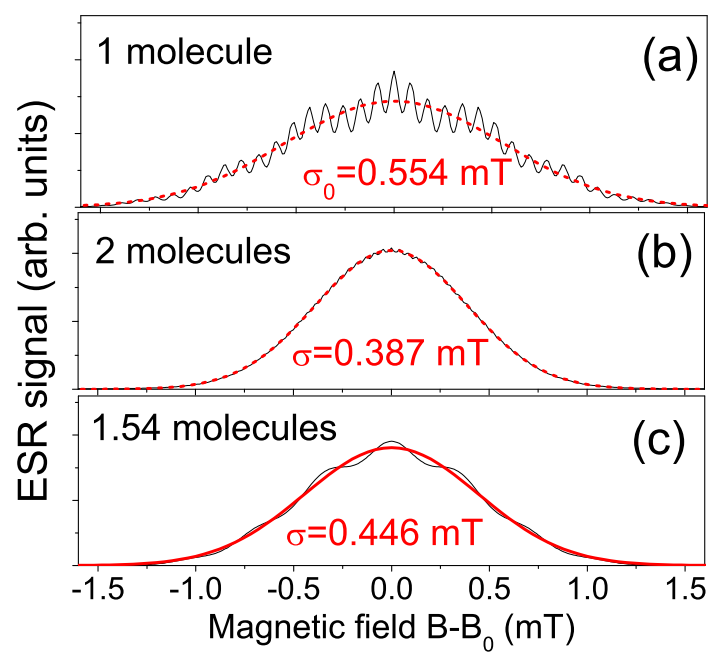

FIG. 1: (color online) Simulated ESR spectrum (black solid lines) of 1,2 , and 1.54 pentacene molecules based on experimental hyperfine splitting of one pentacene molecule with fits by Gauss distribution (red dashed lines).

is given as

$$
P\left(m_{1}, \ldots, m_{k}\right)=\Pi_{i=1}^{k} \frac{C_{2 n_{i} I_{i}}^{m_{i}+n_{i} I_{i}}}{\left(2 I_{i}+1\right)^{-n_{i}}},
$$

where $C_{2 n_{i} I_{i}}^{m_{i}+n_{i} I_{i}}$ are binomial coefficients.

For the particular case of the pentacene molecule we set $\Gamma=0.02 \mathrm{mT}$ and use the coupling constants $\left\{A_{i} ; i=1, \ldots, 4\right\}$ and numbers of equivalent nuclei $\left\{n_{i} ; i=1, \ldots, 4\right\}$ reported in [5]. The ESR signal obtained from Eqs. (11) and (2) can be represented (see Fig. 13) as a curve fluctuating around the Gaussian envelope

$$
G_{0}(B)=\sqrt{\frac{1}{2 \pi \sigma_{0}^{2}}} \exp \left[-\frac{\left(B-B_{0}\right)^{2}}{2 \sigma_{0}^{2}}\right]
$$

with standard deviation $\sigma_{0}=0.554 \mathrm{mT}$.

The standard situation, known from the physics of gases and solutions, is the case where the carrier is localized in a cluster containing $N$ molecules and its density is spread over $N$ molecules. In this case the signal retains its Gaussian shape with the width of the distribution reduced by the factor $N^{1 / 2}$. The hyperfine structure of the $N$ molecules can be simulated by Eqs. (11) and (2) by replacing $n_{i} \rightarrow N n_{i}$ and $A_{i} \rightarrow A_{i} / N$. Figure $1 \mathrm{~b}$ shows an example of the spectrum for $N=2$ with standard deviation $\sigma=\sigma_{0} / \sqrt{2}$. It is clear that the oscillations around the Gaussian envelope are quickly suppressed as $N$ increases.

The shape and the $1 / \sqrt{N}$ narrowing factor of the ESR signal for a carrier distributed over a cluster with $N$ molecules follow from the CLT. The shifts of the signal, $y_{i}=\left(B_{i}-B_{0}\right)$ for the $i$-th molecule, is an independent random variable with Gaussian distribution $R(y)=$ 


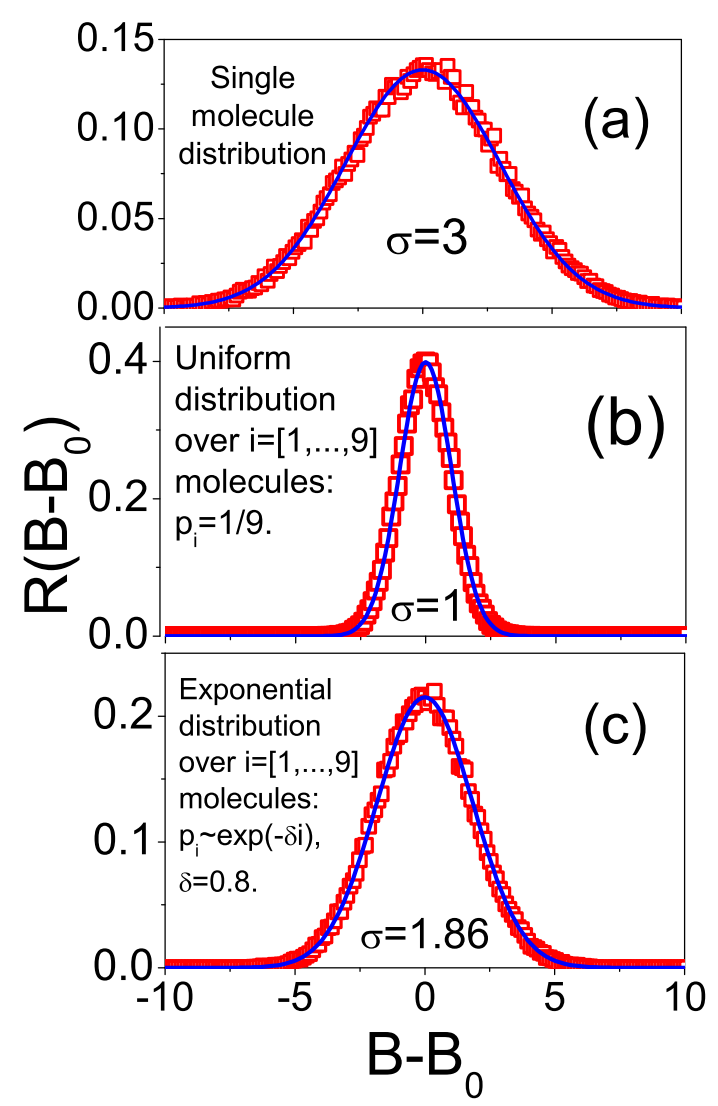

FIG. 2: (color online) (a) Generated Gauss distribution with $\sigma=3$, (b) distribution of a uniformly weighted $p_{i}=1 / 9$ sum of 9 normally distributed random variables, and (c) distribution of exponentially weighted $p_{i}=\exp (-\delta i)(\delta=0.8)$ sum of 9 normally distributed random variables. The squares show the distribution of the generated random variables and the solid lines are the fits by Gaussian functions. The envelope Gaussian function is normalized to unity in all panels.

$G_{0}(y)$ with standard deviation $\sigma_{0}$. By the CLT, the distribution $R(\bar{y})$ of the random variable $\bar{y}=N^{-1} \sum_{i=1}^{N} y_{i}$ is also Gaussian with $\sigma_{N}=\sigma_{0} / \sqrt{N}$.

\section{B. ESR spectra for trap in crystal}

The situation for a carrier localized in a trap in a crystal is different from the above situation with $N$ molecules. The latter case assumes uniform charge distribution, and thus the CLT applies. In contrast, the distribution over molecules $i$ in a trap $\left\{p_{i}\right\}$ is nonuniform with the probabilities $p_{i}$ subjected to the normalization condition $\sum_{i} p_{i} \equiv 1$. Hence, we cannot assume a Gaussian lineshape for the ESR signal; the lineshape must be studied separately.

Regardless of the lineshape, the probability distribution $\left\{p_{i}\right\}$ unambiguously determines the linewidth of the ESR signal. The linewidth is characterized by the stan- dard deviation $\sigma$ which is the root square of the second moment of the linewidth. If we consider the standard deviation of a signal in a trap $\sigma$ and compare it with that from a single molecule $\sigma_{0}$ we can introduce the effective number of molecules $N_{e f f}\left(\left\{p_{i}\right\}\right)$ to describe the linewidth of the ESR signal from a carrier in a trap. The distribution of the ESR shift $B$ is the same for each molecule $i$ with mean $\langle B\rangle=B_{0}$ and variance $\sigma_{0}^{2}=\left\langle\left(B-B_{0}\right)^{2}\right\rangle$. Since the hyperfine configuration of molecules are independent of each other the variables $y_{i}=\left(B_{i}-B_{0}\right)$ are independent for different molecules $i$. Hence, the standard deviation $\sigma\left(\left\{p_{i}\right\}\right)$ of the sum of random variables $\bar{y}=\sum_{i} p_{i}\left(B_{i}-B_{0}\right)$ is related to the single-molecule standard deviation $\sigma_{0}$ by the expression

$$
\frac{\sigma\left(\left\{p_{i}\right\}\right)}{\sigma_{0}}=\sqrt{\sum_{i} p_{i}^{2}} .
$$

It is natural to define the effective number of molecules $N_{\text {eff }}\left(\left\{p_{i}\right\}\right)$, corresponding to the charge distribution $\left\{p_{i}\right\}$, to be

$$
\sigma\left(\left\{p_{i}\right\}\right)=\sigma_{0} / \sqrt{N_{e f f}\left(\left\{p_{i}\right\}\right)} .
$$

Then, the effective number of molecules $N_{e f f}\left(\left\{p_{i}\right\}\right)$ is unambiguously determined by the charge distribution in a trap $\left\{p_{i}\right\}$

$$
N_{\text {eff }}\left(\left\{p_{i}\right\}\right)=\left[\sum_{i} p_{i}^{2}\right]^{-1} .
$$

To study the shape of the ESR signal for a trap with charge density $\left\{p_{i}\right\}$ we generated by a standard method ${ }^{24}$ random variables $\left\{y_{i}\right\}$ following a Gaussian distribution with dispersion $\sigma_{0}=3$ (Fig. 2a). Then, we studied the distribution of the random variable $\bar{y}=\sum_{i} p_{i} y_{i}$. By the CLT the uniform distribution $p_{i}=1 / N$ leads to a Gaussian shape of the signal with the dispersion narrowed by the factor $\sqrt{N}$ (Fig. 2b). After performing simulations with a large set of different distributions $p_{i}$ we conclude that the distribution of the random variable $\bar{y}=\sum_{i} p_{i} y_{i}$ is always Gaussian (see Fig. 2r) with $N_{e f f}\left(\left\{p_{i}\right\}\right)$ defined by Eq. (6).

Hence, we conclude that the shape of the ESR signal for carriers localized in a set of identical independent traps is uniquely determined by the distribution density $\left\{p_{i}\right\}$. It is always Gaussian with the standard deviation $\sigma$ defined by Eqs. (5) and (6).

\section{ESR spectra for several kinds of traps}

Since the ESR signal for independent identical traps is always Gaussian, we assume that a non Gaussian shape originates from the superposition of the signals from different kinds of traps. Indeed, each different kind of trap is characterized by a different probability distribution of the trapped carriers. 
To describe the experimental spectrum $\mathcal{E}_{\text {exp }}(B)$ by the superposition of the ESR spectra $\mathcal{G}(B, \xi)$ for each trap type $\xi$ we must choose a parameter $\xi$ that unambiguously characterizes the spectrum $\mathcal{G}(B, \xi)$. It follows from the analysis in Section $\amalg A$ that the ESR spectrum from identical traps is Gaussian and can be characterized by a single parameter $N_{\text {eff }}$. This parameter reflects the spatial distribution $\left\{p_{i}\right\}$ of a charge in a trap (6) and determines the narrowing of the Gaussian $\mathcal{G}\left(B, \xi=N_{\text {eff }}\right)$ width $\sigma\left(\left\{p_{i}\right\}\right)=\sigma_{0} / \sqrt{N_{e f f}\left(\left\{p_{i}\right\}\right)}$ with respect to the ESR width $\sigma_{0}$ of a carrier localized on a single molecule. Therefore, the ESR signal for the same trap type, characterized by the same spatial extension $\xi=N_{e f f}$, can be expressed as

$$
G\left(B, N_{e f f}\right)=\sqrt{\frac{N_{e f f}}{2 \pi \sigma_{0}^{2}}} \exp \left[-\frac{\left(B-B_{0}\right)^{2}}{2\left(\sigma_{0}^{2} / N_{e f f}\right)}\right] .
$$

Introducing the distribution function $D\left(N_{e f f}\right)$ of the traps in pentacene over $N_{\text {eff }}$ we can express the experimental signal $\mathcal{E}_{\text {exp }}(B)$ in terms of the superposition

$$
\mathcal{E}_{\text {exp }}(B)=\int_{1}^{\infty} G\left(B, N_{e f f}\right) D\left(N_{e f f}\right) d N_{e f f}
$$

which is a convolution of the distribution function $D\left(N_{e f f}\right)$ of the traps and the Gaussian signal for a trap type characterized by the localization parameter $N_{\text {eff }}$.

Most techniques for ESR measurements detect the derivative $\mathcal{E}_{\exp }(B)$ over the magnetic field $B$ and hence the experimental signal $\mathcal{X}_{\exp }(B)=d \mathcal{E}_{\exp }(B) / d B$ is related to the distribution function of the traps $D\left(N_{\text {eff }}\right)$ via

$$
\mathcal{X}_{\text {exp }}(B)=\int_{1}^{\infty} \frac{d G\left(B, N_{e f f}\right)}{d B} D\left(N_{e f f}\right) d N_{e f f} .
$$

Hence, to obtain the distribution $D\left(N_{\text {eff }}\right)$ we must solve one of the integral equations (8) and (91). The experimental signals $\mathcal{X}_{\exp }(B)\left(\mathcal{E}_{\exp }(B)\right)$ and the kernel $d G\left(B, N_{e f f}\right) / d B\left(G\left(B, N_{e f f}\right)\right)$ are known functions and the distribution $D\left(N_{e f f}\right)$ is to be determined.

\section{FROM ESR SPECTRUM TO TRAP DISTRIBUTION: CONVERSION OF EXPERIMENTAL LINESHAPE INTO DISTRIBUTION OF TRAPS OVER DEGREE OF LOCALIZATION}

Equation (8) [(9)], where $\mathcal{E}_{\exp }(B) \quad\left[\mathcal{X}_{\exp }(B)\right]$ and $G\left(B, N_{e f f}\right)\left[d G\left(B, N_{e f f}\right) / d B\right]$ are known and $D\left(N_{e f f}\right)$ is to be determined, is a Fredholm equation of the first kind. Naively, to find the solution $\widetilde{D}\left(N_{\text {eff }}\right)$ we must maximize the inverse deviation

$$
Q=\left\{\int_{B_{\min }}^{B_{\max }}\left|\mathcal{X}_{\exp }(B)-\widetilde{\mathcal{X}}(B)\right|\right\}^{-1} .
$$

Here $B_{\min }\left(B_{\max }\right)$ is the lower (upper) bound of a magnetic field where the signal is larger than the experimental noise. $\mathcal{X}_{\text {exp }}(B)$ are experimental data and $\widetilde{\mathcal{X}}(B)$ is obtained from the distribution $\widetilde{D}\left(N_{e f f}\right)$

$$
\widetilde{\mathcal{X}}(B)=\int_{0}^{\infty} \frac{d G\left(B, N_{e f f}\right)}{d B} \widetilde{D}\left(N_{e f f}\right) d N_{e f f}
$$

which is considered to be a solution of the integral equation. However, such naive approach leads to huge unrealistic oscillations of the solution $\widetilde{D}\left(N_{\text {eff }}\right)$. Instead, we need to apply one of the advanced techniques developed for such equations.

Section $\amalg I$ A gives a general description of the stochastic optimization method $(\mathrm{SOM})^{24-27}$ for the solutoion of Eqs. (8) and (9). Section IIIB applies the method to the analysis of the ESR data and demonstrates the influence of experimental noise on the reliability of the results. Section IIIC presents an experimental technique to obtain ESR spectra suitable for the fine analysis of the data. Section IIID introduces an algorithm that implements the SOM and presents results for the trap distribution in pentacene TFTs. Finally, Section ஹIE demonstrates the limits of the reliability of the distribution obtained by solving Eqs. (8) and (9).

\section{A. Method to solve inverse problem}

It is notoriously difficult to solve the Eqs. (8) and (9) because these equations belong to the class of "ill posed" problems. Naively, the true solution of the Eq. (9) $\widetilde{D}\left(N_{e f f}\right)$, being convoluted with the kernel, produces the function $\tilde{\mathcal{X}}(B)$, - which coincides with the given function $\mathcal{X}_{\text {exp }}(B)$. However, the general feature of the practical implementations of Eq. (9) is that the knowledge about the function $\mathcal{X}_{\exp }(B)$ is "noisy" and incomplete. Specifically, $\mathcal{X}_{\text {exp }}(B)$ is known for a particular set of points $\left\{B_{i}, i=1, M\right\}$ with some errorbars resulting from the experimental noise. In this case, to find a "solution", we can introduce the residual function

$$
\Delta(i)=\tilde{\mathcal{X}}\left(B_{i}\right)-\mathcal{X}_{\exp }\left(B_{i}\right), \quad i=1, M
$$

and optimize a measure of the deviation of "the solution" $\tilde{\mathcal{X}}(B)$ from the given data $\mathcal{X}_{\exp }(B)$. For example, we could maximize the inverse deviation (10)

$$
Q=\left[\sum_{i=1}^{M}|\Delta(i)|\right]^{-1}
$$

Naturally, $\Delta(i)$ is never equal to zero at all points $i=$ $1, M$ when realistic noisy data $\left\{\mathcal{X}_{\exp }\left(B_{i}\right), i=1, M\right\}$ are considered. Hence, even the best measure $Q^{\max }$ is not equal to infinity. Therefore, the only feasible strategy for Eq. (9) is to find a solution, that is "the best" in some sense. 
The above features are the characteristics of the class of "ill posed" problems for which we can not get an exact solution and can only find the "best" choice of $\widetilde{D}\left(N_{e f f}\right)$ for the given data set $\left\{\mathcal{X}_{\text {exp }}\left(B_{i}\right), i=1, M\right\}$. The naive approach, where we simply maximize measure (13), leads to unreasonable "solutions". Typically, they have huge fluctuations which exceed the true values of $D\left(N_{e f f}\right)$ by several orders of magnitude. To get a reasonable description of $D\left(N_{e f f}\right)$ we must suppress this "saw tooth" instability.

There are two different strategies. The first is the regularization method, e.g. the popular maximal entropy $\operatorname{method}^{34}$ as one of many such methods ${ }^{35,36}$. This approach maximizes a measure that is similar to (13) but modified in such a way that the solution is smooth enough to suppress "saw tooth noise". The main drawback is that the solution is corrupted by the smoothing regularization procedure. The second strategy uses modern stochastic approaches to obtain many statistically independent solutions ( $\sec ^{24,27}$ and the references therein) whose linear combination smoothes the "saw tooth noise" without corrupting individual solutions. Since this approach has been shown 27 to be better for the solution of Eq. (9), we applied the $\mathrm{SOM}^{24-26}$ as a particular example of the stochastic technique ${ }^{27}$.

The function $D\left(N_{\text {eff }}\right)$ is a distribution function and, hence, it is non negative $D\left(N_{\text {eff }}\right) \geq 0$ and normalized

$$
\int_{a}^{b} D\left(N_{e f f}\right) d N_{e f f}=I
$$

to a certain number $I$. The noise present in experimental data $\left\{\mathcal{X}_{\text {exp }}\left(B_{i}\right), i=1, M\right\}$ makes the problem of normalization nontrivial. Some approaches to handle the normalization problem are suggested in the next chapters.

\section{B. Tests of SOM and role of noise in experimental data}

The SOM has been successfully applied to many integral equations. The kernels of the equations are different from those in Eqs. (8) and (91). The exponential kernel $K(y, x)=\exp [-y x]$ was examined in $24,37,55]$, and various kernels ranging from the Fermi distribution to the Matsubara frequency representation are considered in [56 59]. We test the applicability of the SOM to the kernels of Eqs. (8) and (9). We also show how the statistical noise of the experimental data can obscure the information that can otherwise be obtained by solving these equations.

To verify that the SOM is applicable to Eqs (8) and (9) with the kernel defined by (7) we introduced a normalized to unity function $D\left(N_{e f f}\right)$ (see the dotted line in Figs. 3 and (4) and generated a set of "experimental" data $\left\{\mathcal{X}_{\text {exp }}\left(B_{i}\right), i=1,200\right\}$ using relations (819). Then, we attempted to find $\widetilde{D}\left(N_{e f f}\right)$ by solving Eqs. (8) and (9). For ideal data without noise in the set

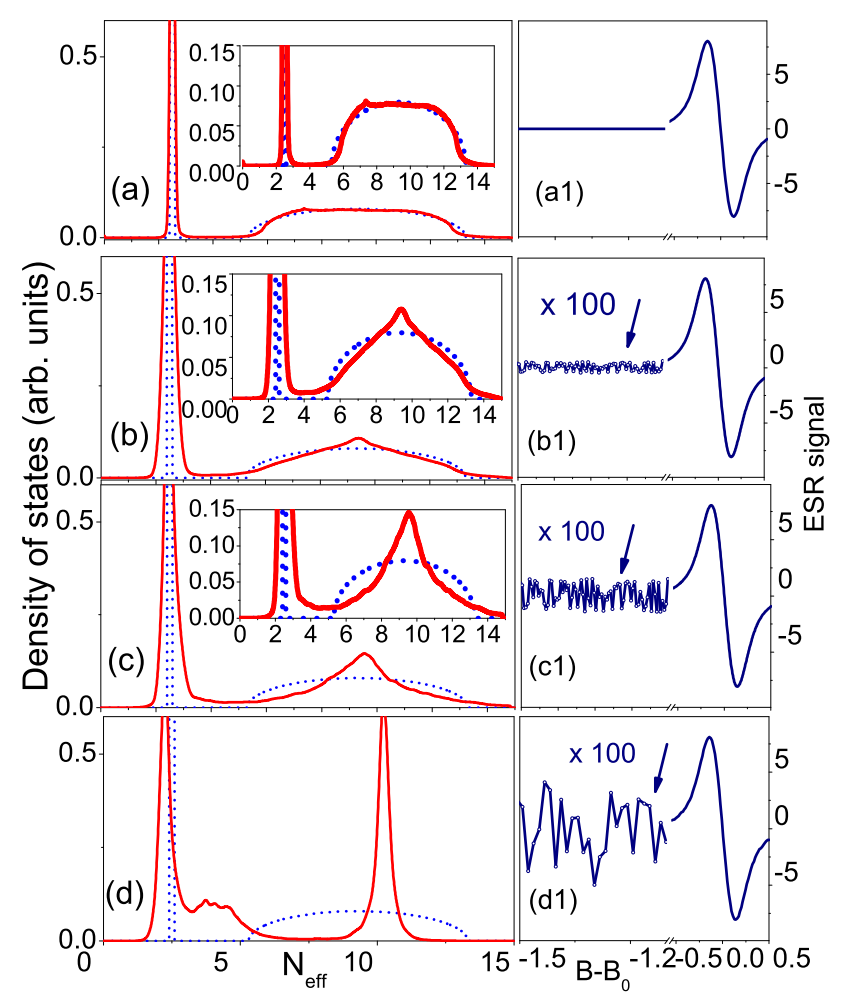

FIG. 3: (color online) Test of SOM procedure to restore distribution of spatial extent of traps $D\left(N_{e f f}\right)$ from "experimental" ESR data $\mathcal{X}_{\exp }(B)$ (9) under different levels of noise. "Experimental" ESR data with noise are given in panels (a1)-(d1) and actual (restored) spectrum $D(x)$ is indicated by dashed (solid) line in corresponding panels (a)-(d). Noise level is (a,a1) $f=0.0,(\mathrm{~b}, \mathrm{~b} 1) f=0.01\left[s_{n}=10^{-3}\right],(\mathrm{c}, \mathrm{c} 1) f=0.03$ $\left[s_{n}=3 * 10^{-3}\right]$, and $(\mathrm{d} 1, \mathrm{~d}) f=0.1\left[s_{n}=10^{-2}\right]$. Restored spectrum is obtained by solving Eq. (9) using SOM. Insets show details of corresponding spectra.

$\left\{\mathcal{X}_{\exp }\left(B_{i}\right), i=1,200\right\}$ we were able to restore $D\left(N_{\text {eff }}\right)$ successfully (see Fig. 3(a) and 4(a)). We did not find a significant difference between the results obtained by solving Eqs. (8) and (9).

In spite of the extensive usage of $\mathrm{SOM}^{24,37-59}$ there is no precise understanding of how the errorbars of the data $\left\{\mathcal{X}_{\text {exp }}\left(B_{i}\right), i=1, M\right\}$ obscure the solution $\widetilde{D}\left(N_{\text {eff }}\right)$. The difficulty is the dependence of the result $\widetilde{D}\left(N_{e f f}\right)$ on the level of statistical noise, the shape of $D\left(N_{e f f}\right)$, the kernel, and even the number of points $M$ in the data set $\left\{\mathcal{X}_{\exp }\left(B_{i}\right), i=1, M\right\}$. Therefore, below we present some examples showing how the noise of the experimental data obscures the solution of Eq. (9). These ad hoc examples indicate the general trends but must not be treated as a quantitative analysis of the influence of noise on the reliability of the SOM results. These data should not be used for the analysis of other cases. In Section IIIE we present a procedure to check the reliability of particular solutions.

To introduce noise we used a sequence of random numbers $\mathcal{R}(i)$ uniformly distributed in the range $[-1,1]$ and 


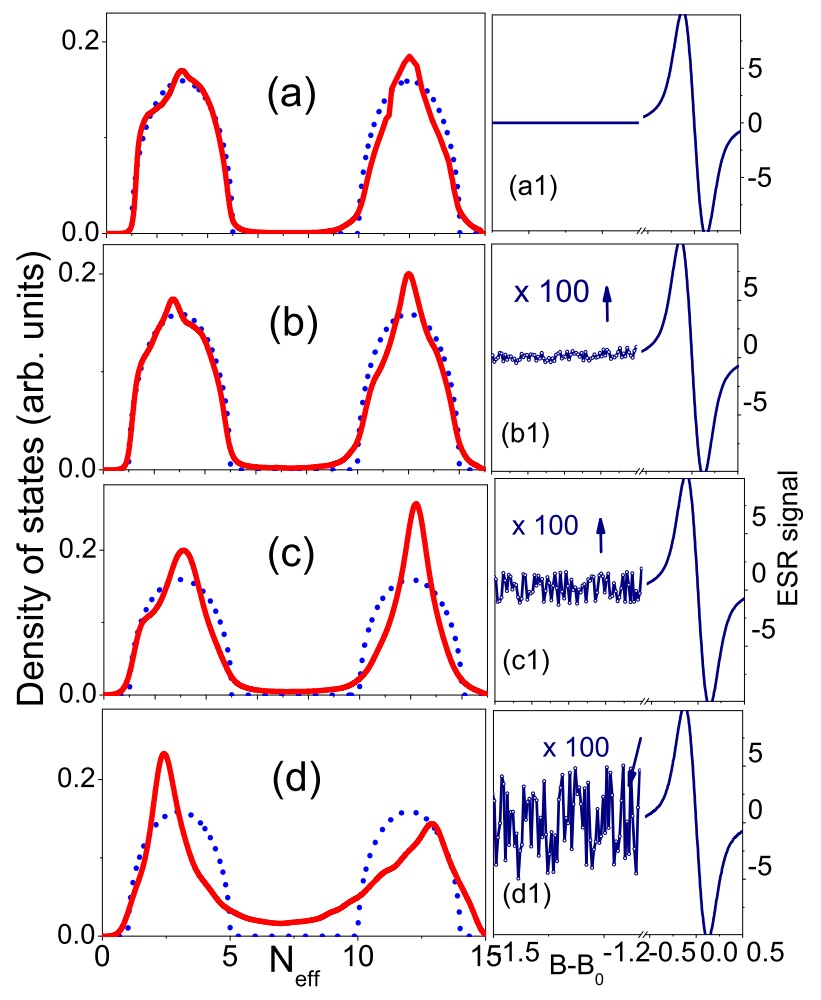

FIG. 4: (color online) See figure caption in Fig. 3 .

generated data sets $\left\{\mathcal{X}_{\text {exp }}\left(B_{i}\right)+(f / 2) \mathcal{R}(i), i=1,200\right\}$, referred in the following as "experimental" ESR data, with different amplitudes of the noise $f$. The signalto-noise ratio is defined as a ratio of the amplitude $f$ and maximal absolute value $M A X\left\{\left|\mathcal{X}_{\exp }\left(B_{i}\right)\right|\right\}$ of the given signal $\mathcal{X}_{\text {exp }}\left(B_{i}\right)$ : as $s_{n}=f / M A X\left\{\left|\mathcal{X}_{\text {exp }}\left(B_{i}\right)\right|\right.$. The results presented in Figs 3 and 4 illustrate the general trends. An increase in the signal-to-noise ratio $s_{n}$ corrupts the solution for large values of $N_{\text {eff }}$ first: the shape of the high-energy peak is not reproduced but its position is still correct. At higher values of $s_{n}$ the shape of the low-energy peak is not reproduced. A comparison of Figs 3 and 4 shows that the spectrum with a sharp feature at small $N_{\text {eff }}$ (Fig. 3i) is more robust to experimental noise than that with a broad feature at small $N_{\text {eff }}$ (Fig. 4). Note that although the shapes of the high- and low-energy peaks are not reproduced, their positions are still approximately correct even for large values of the signal-to-noise ratio $s_{n}$.

\section{Experimental data for analysis}

To conduct reliable spectral analysis as discussed above, we need high-precision ESR spectra for the carriers in organic TFTs. We acquired the spectra by the following procedures: We used a commercially-available X-band (9 GHz) ESR apparatus (JES-FA200, JEOL) equipped with a high Q cylindrical cavity (Q factor 4000-
6000 for the $\mathrm{TE}_{011}$ mode). We fabricated bottom-gate, top-contact pentacene TFTs with high mobility that are suitable for high-precision measurements. The device is composed of a 100- $\mu \mathrm{m}$-thick poly(ethylene naphthalate) (PEN) film as a nonmagnetic substrate, a 1- $\mu$ m-thick Parylene C film as a gate dielectric layer $\left(4.5 \mathrm{nF} / \mathrm{cm}^{2}\right)$, and a 50-nm-thick pentacene film as the semiconducting layer. The gate, source, and drain electrodes are composed of vacuum-deposited gold films with a thickness of $30 \mathrm{~nm}$; this is much thinner than the skin depth of gold (about $790 \mathrm{~nm}$ ).

Since the field-induced carrier is accumulated only at the semiconductor/insulator interface, the ESR signal is proportional to the total channel area of the TFTs. We used a device with a width of $2.5 \mathrm{~mm}$ and a length of $20 \mathrm{~mm}$, the dimension of which is limited by the inner diameter of the ESR tube and the cavity size. We used a stack of ten sheets of TFTs for the high-precision ESR measurement, to obtain field-induced carriers ten times as large as those in a single sheet. The total carrier number at $V_{G}=-200 \mathrm{~V}$ is estimated as $2.8 \times 10^{13}$.

The semiconducting pentacene layer is composed of a uniaxially-oriented polycrystalline film where all the component pentacene molecules aligned with the molecular long axes are roughly perpendicular to the film plane. In the measurement, a static magnetic field was set perpendicular to the film plane to eliminate the anisotropic effect of the $\mathrm{g}$ tensor. A continuous-flow cryostat was used for the low-temperature measurements. In the FESR measurements at low temperature, we first applied the gate voltages at room temperature (with the source and drain shorted) and then cooled the device to the set temperature, to avoid a delay in the charge accumulation. The temperature was stabilized carefully so that the fluctuation at $20 \mathrm{~K}$ was about $0.01 \mathrm{~K}$, which minimized the effect of temperature-dependent spin susceptibility.

\section{Practical implementation of method: Distribution of traps in pentacene TFT}

Equation (9) is preferable for the practical implication of the SOM. The problem with realistic noisy data from ESR experiments is that there is some uncertainty in the normalization and background of the experimental data. In the ideal case, implying the normalization of the distribution $D\left(N_{e f f}\right)$ to unity and the conditions $\int_{-\infty}^{\infty} G\left(B, N_{e f f}\right) d N_{\text {eff }}=1$ and $\int_{-\infty}^{\infty} d N_{\text {eff }} \int_{-\infty}^{B} d z\left[d G\left(z, N_{e f f}\right) / d z\right]=1$, we must normalize the experimental data as

$$
\int_{-\infty}^{\infty} d B \mathcal{E}_{\exp }(B)=1
$$

and

$$
\int_{-\infty}^{\infty} d B \int_{-\infty}^{B} d z \mathcal{X}_{\exp }(z)=1
$$




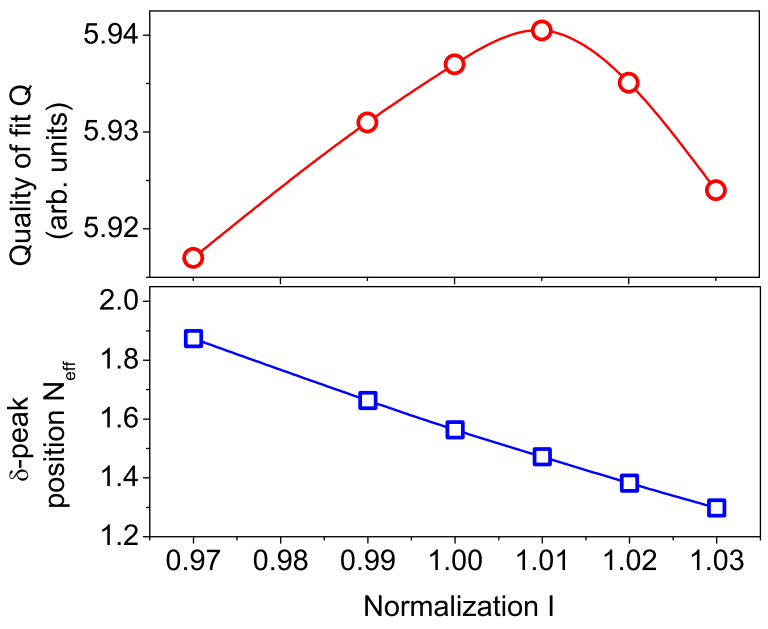

FIG. 5: (color online) (a) Dependence of the fit quality $Q^{\max }$ and position of low-energy peak $\delta\left(N-N_{e f f}\right)$ on normalization $I$ of the spectral function.

respectively. However, the normalization of the experimental data is not exact because experimental noise can lead to uncertainty of a few percentage points. On the other hand, the solution of Eq. (9) is sensitive to possible normalization errors.

There is also a problem with the background. If the experimental data are obtained in the form $\mathcal{E}_{\exp }(B)$ (see e.g. Fig. (2), there is no unique procedure to determine the constant background level that must be subtracted from the data to get a pure signal for the ESR transition. This uncertainty also increases the uncertainty relating to the normalization of the data. However, the problem of the unknown background disappears when Eq. (9) is used as the integral equation because $\lim _{B \rightarrow \pm \infty} \mathcal{X}_{\text {exp }}(B)=0$ for any constant background. Therefore, to analyze the ESR data for pentacene TFTs we used (9) where the only uncertainty is that of normalization.

To handle the normalization uncertainty we can change the normalization in either Eq. (14) or Eq. (16). The two choices are equivalent because of the linearity of Eq. (9). In practice we normalized the experimental signal as shown in (16) and varied the normalization $I$ of the distribution density $\widetilde{D}\left(N_{\text {eff }}\right)$ (14). We handled the normalization uncertainty for the result shown in Fig. 6] as follows. The spectrum at gate voltage $-200 \mathrm{~V}$ was considered (the result for this gate voltage is shown in Fig. 6 by solid line). The integral equation (9) was solved for different normalizations $I$ and the solution with the normalization having the best deviation measure $Q^{\max }$ was chosen.

Figure 5 shows the best inverse deviation $Q^{\max }$ (10]13) and the position of the sharp peak in $D\left(N_{e f f}\right)$ versus the normalization $I$. It can be seen that the position of the sharp peak is sensitive to the value of the normalization $I$ and this may be a source of the volatility in the fine

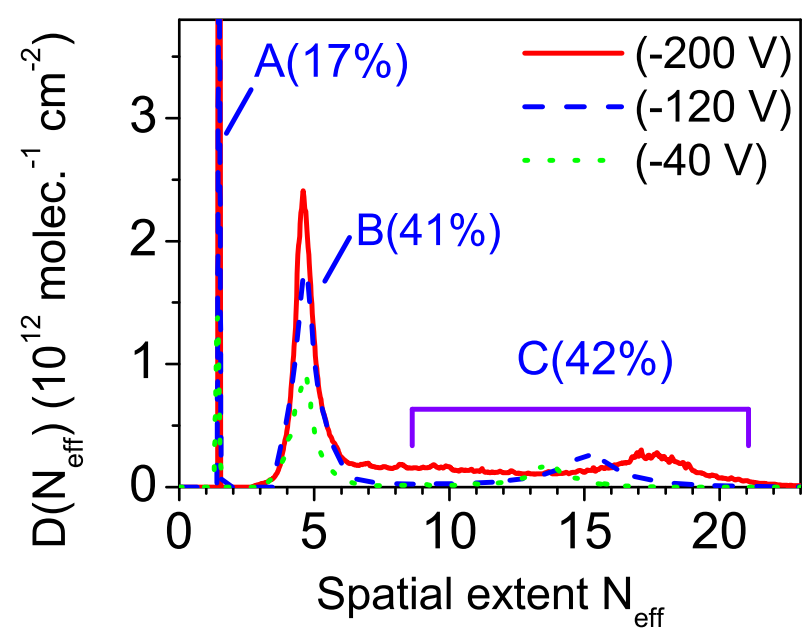

FIG. 6: (color online) Distribution of trap states in pentacene TFT versus spatial extent $N_{\text {eff }}$ of charge distribution in traps obtained from ESR spectrum of pentacene TFT at $20 \mathrm{~K}$ with gate voltage $-200 \mathrm{~V}$ (solid line), $-120 \mathrm{~V}$ (dashed line), and -40 $\mathrm{V}$ (dotted line).

analysis of the ESR data. However, it can be shown that the suggested approach to the the normalization $I$, which leads to the best deviation measure $Q^{\max }$, is robust and produces stable results. We have demonstrated this via analysis of the ESR data at different gate voltages (Fig. 6). We found that the best normalizations are different at different gate voltages: $I(V=-200)=1.01$, $I(V=-120)=1.038$, and $I(V=-40)=1.03$, respectively. However, the position of the sharp peak at low values of $N_{\text {eff }}$ does not depend on the voltage $V$ if at each voltage $V$ we use the normalization $I(V)$ that corresponds to the best deviation measures $Q^{\max }$. Physically, the sharp peak at low values of $N_{\text {eff }}$ corresponds to deep impurity levels that depend only slightly on the gate voltage. Therefore, its independence on the gate voltage in the fine analysis of the ESR spectra indicates the high stability of the procedure based on the suggested approach.

\section{E. Reliability of trap distribution result}

Since solving Eq. (9) is an "ill-posed" problem it is useful to understand how much information we can get from the analysis and to check how many details of the resulting distribution of impurities are reliable. The reliability can be analyzed by plotting the residual function (12).

The spectrum $\widetilde{D}\left(N_{\text {eff }}\right)$ (Fig. 6) obtained by solving Eq. (9) has three peaks. Figure. 7h shows the fit of the ESR signal using the distribution $\widetilde{D}\left(N_{e f f}\right)$ in Fig. 6] It also shows the separate contributions of the $\mathrm{A}, \mathrm{B}$, and $\mathrm{C}$ 


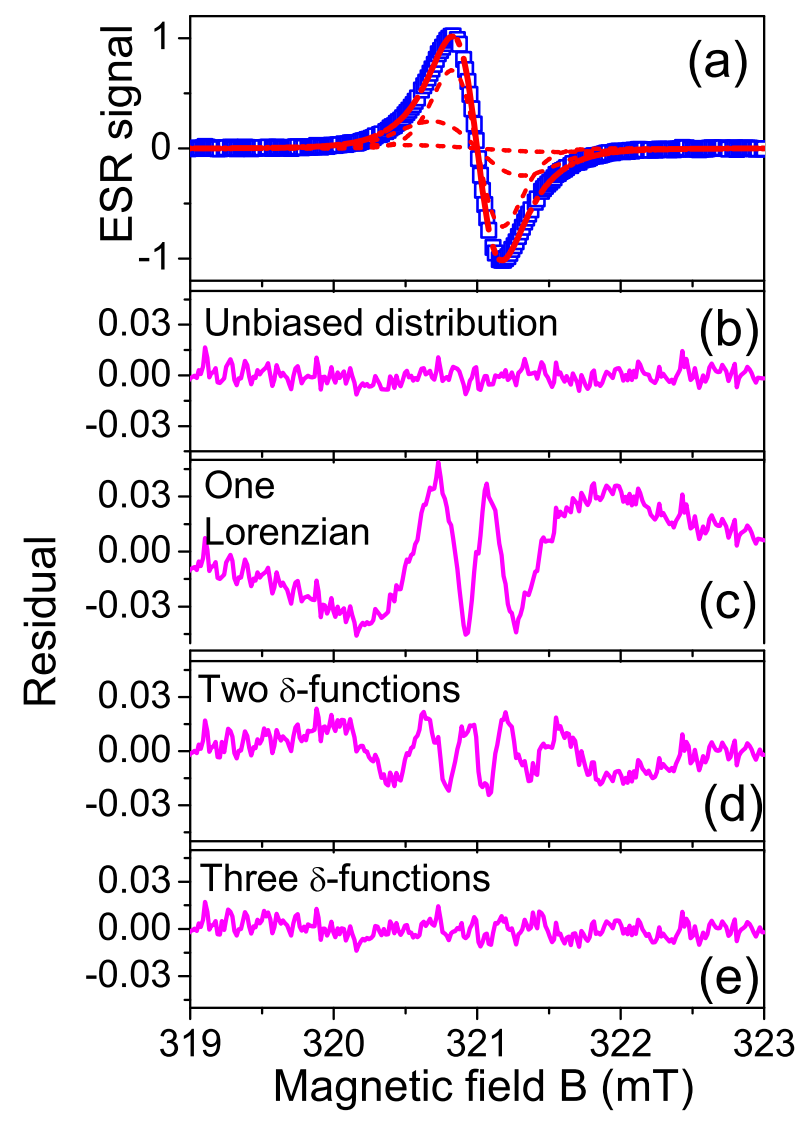

FIG. 7: (color online) (a) Experimental signal (squares), fit by spectrum in Fig. 6] at the gate voltage $-200 V$ (solid lines) and contributions from (see Fig. 6) A, B, and C components (dashed lines). Residuals (12) $\mathcal{X}_{\text {exp }}(B)-\widetilde{\mathcal{X}}(B)$ from (b) unbiased distribution (Fig. 6), (c) best fit by one Lorentzian, (d) best fit by two $\delta$-functions $0.68 \delta(N-4.5)$ and $0.32 \delta(N-20)$, and (e) best fit by three $\delta$-functions $0.31 \delta(N-1.4), 0.51 \delta(N-7.5)$, and $0.18 \delta(N-25.0)$.

components of the distribution. To clarify which features of the distribution $\widetilde{D}\left(N_{e f f}\right)$ are reliable for the given level of noise in the experimental data we studied the residuals (12) $\mathcal{X}_{\text {exp }}(B)-\widetilde{\mathcal{X}}(B)$ (Figs. 7b-e). We can see that the quality of the fit by the SOM (Fig. 7b) is much better than that obtained by e.g., the Lorentzian (Fig. 7 F) and two $\delta$-functions (Fig. $7 \mathrm{~d}$ ). The fit from three $\delta$-functions gives a residual function (Fig. 7f) as good as that obtained from $\widetilde{D}\left(N_{e f f}\right)$ in Fig. 6. Therefore, we conclude that, within the limits of the noise of the experimental data, the existence of at least three kinds of traps is a reliable result.

We note that the distribution over the parameter $N_{\text {eff }}$ in Fig. 6 is free from any assumption about the shape of the distribution. Indeed, because of the noise of the current experimental data, the only reliable conclusion is the statement about the existence of at least three types of traps. However, a data analysis with less noise could, in principle, reveal more fine structure in the distribution function $D\left(N_{\text {eff }}\right)$.

\section{TRANSFORMATION FROM SPATIAL DISTRIBUTION TO ENERGY DISTRIBUTION}

In this section we discuss finding the distribution of the traps $\mathcal{Z}\left(E_{B}\right)$ over the binding energies $E_{B}$ given the distribution $D\left(N_{e f f}\right)$ over the localization parameter $N_{\text {eff }}$. This transformation is trivial when there is $a$ priori knowledge of the functional dependence

$$
N_{\text {eff }}=N_{\text {eff }}\left(E_{B}\right) .
$$

Indeed, there is a balance relation

$$
\begin{gathered}
\mathcal{Z}\left(\frac{E_{B}^{(i+1)}+E_{B}^{(i)}}{2}\right)\left[E_{B}^{(i+1)}-E_{B}^{(i)}\right]= \\
D\left(\frac{N_{e f f}\left(E_{B}^{(i+1)}\right)+N_{e f f}\left(E_{B}^{(i)}\right)}{2}\right) \\
{\left[N_{\text {eff }}\left(E_{B}^{(i+1)}\right)-N_{\text {eff }}\left(E_{B}^{(i)}\right)\right]}
\end{gathered}
$$

between $D$ and $\mathcal{Z}$ for two nearby points $E_{B}^{(i+1)}$ and $E_{B}^{(i)}$. Then, in the limit $E_{B}^{(i+1)} \rightarrow E_{B}^{(i)}$ we get

$$
\mathcal{Z}\left(E_{B}\right)=D\left(N_{e f f}\right) \frac{d N_{e f f}\left(E_{B}\right)}{d E_{B}}
$$

The above generic relation (17) can be obtained as a parametric function provided we can to choose an appropriate model and calculate $N_{\text {eff }}=N_{\text {eff }}(\{\mathcal{P}\})$ and $E_{B}=E_{B}(\{\mathcal{P}\})$ in some domain $\{\mathcal{P}\}$ of the parameters $\mathcal{P}$.

The general strategy for the transformation $D \rightarrow \mathcal{Z}$ does not depend on the model. However, the specific form of relation (17) depends on the model chosen to describe the localization of a carrier in a trap. To study carrier trapping in pentacene TFTs we consider a model of a two-dimensional (2D) Holstein polaron in a field of an attractive center. This model is considered in Section IVA and the results of the transformation for pentacene TFTs are given in Section IVB

\section{A. Model for traps: 2D Holstein polaron in field of on-site attractive center}

It is already well known that the behavior of a carrier in pentacene TFTs can be described by a particle in a system with attractive impurities 10 . It is also known that the carrier is subject to the electron-phonon interaction $\underline{\underline{60}}$. To model the behavior of a carrier in pentacene TFTs we chose the simplest Hamiltonian describing a 2D Holstein polaron in a field of on-site attractive center

$$
\begin{gathered}
H=-t \sum_{\langle\mathbf{i} \mathbf{j}\rangle} c_{\mathbf{i}}^{\dagger} c_{\mathbf{j}}+\omega_{\mathrm{ph}} \sum_{\mathbf{i}} b_{\mathbf{i}}^{\dagger} b_{\mathbf{i}} \\
-\gamma \sum_{i}\left(b_{\mathbf{i}}^{\dagger}+b_{\mathbf{i}}\right) c_{\mathbf{i}}^{\dagger} c_{\mathbf{i}}-U c_{\mathbf{0}}^{\dagger} c_{\mathbf{0}}
\end{gathered}
$$




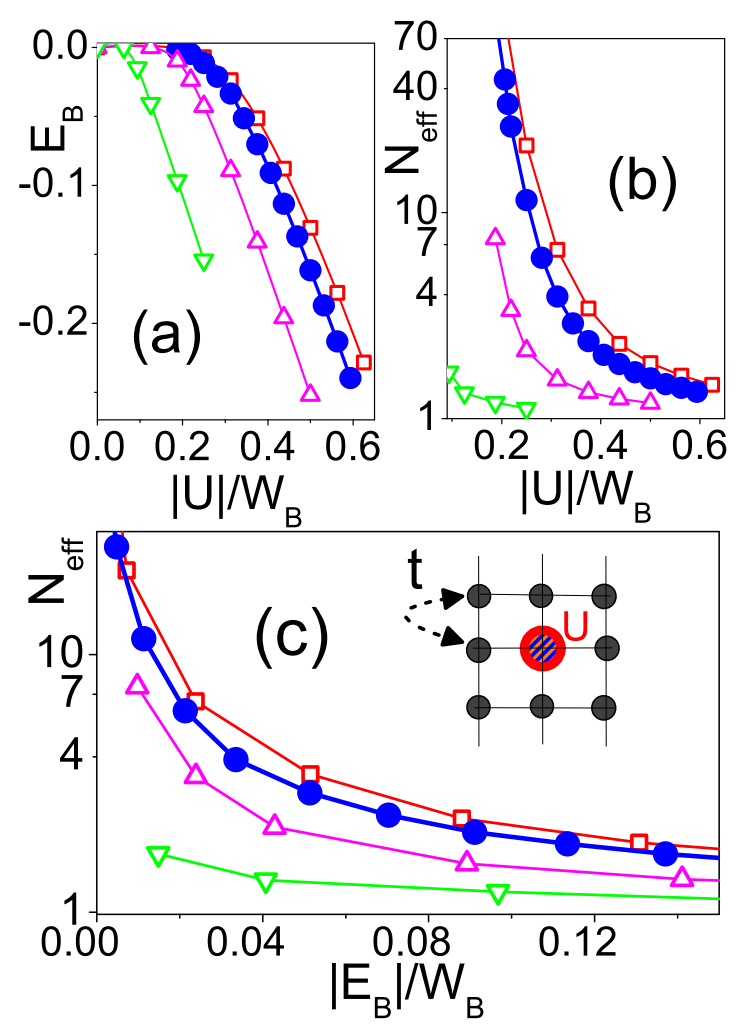

FIG. 8: (color online) Dependence of (a) binding energy $E_{B}$ in units of bandwidth $W_{B}=8 t$ and (b) effective number of molecules $N_{\text {eff }}$ on absolute value of potential of attractive impurity $|U|$ in units of $W_{B}$. (c) Dependence of effective localization number $N_{e f f}$ on binding energy $E_{B}$ in units of bandwidth $W_{B}$. Curves are presented for $\lambda=0$ (squares), $\lambda=0.15$ (circles), $\lambda=0.5$ (triangles pointing up), and $\lambda=1$ (triangles pointing down). Lines are to guide the eye. Inset in (c) shows schematically model represented by Hamiltonian (19).

Here, $c_{i}^{\dagger}\left(b_{i}^{\dagger}\right)$ is the creation operator for the carrier (phonon) in the i-th molecule. $U$ is the attractive impurity potential for the carrier $c_{0}^{\dagger}$ at site 0 and $\omega_{\text {ph }}$ is the frequency of the dispersionless phonon. The amplitude $t$ describes the electron transfer $\propto t$ between nearest neighbor sites and the local Holstein coupling to the phonons is $\propto \gamma$. The dimensionless electron-phonon coupling constant $\lambda$ is defined to be $\lambda=\gamma^{2} /\left(4 t \omega_{\mathrm{ph}}\right)$. It is clear that for the chosen model the parameter set to determine relation (17) is $\mathcal{P}=\{U, \lambda\}$ including the potential of the attracting trap $U$ and the strength of the electron-phonon coupling $\lambda$.

To calculate the values of $E_{B}=E_{B}(U, \lambda)$ and $N_{\text {eff }}=$ $N_{\text {eff }}(U, \lambda)$ we used the direct space diagrammatic Monte Carlo (DSDMC) technique $\stackrel{28}{\underline{2}}$. Similar data can be can be obtained by the inhomogeneous momentum average approximation method ${ }^{29,30}$ and the coherent basis states method 31,32 . The data for $E_{B}=E_{B}(U, \lambda)$ and $N_{\text {eff }}=N_{\text {eff }}(U, \lambda)$ are presented in Fig. $8 \mathrm{a}$ and $8 \mathrm{~b}$. The values of $N_{\text {eff }}$ were determined by relation (6) from the

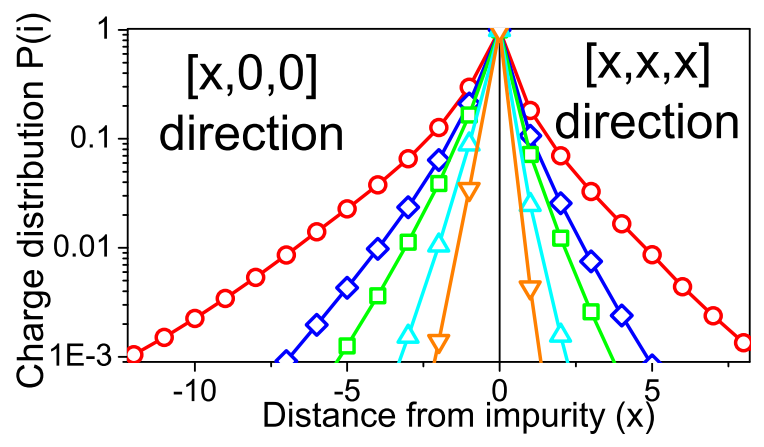

FIG. 9: (color online) Charge distributions around attractive impurity with potential $U$ at $\lambda=0.15$ along [100] and [111] directions: $U / W_{B}=-0.206\left[E_{B} / W_{b}=0.0032, N_{\text {eff }}=44.3\right]$ (circles); $U / W_{B}=-0.25\left[E_{B} / W_{b}=0.0113, N_{\text {eff }}=11.2\right]$ (rhombus); $U / W_{B}=-0.281\left[E_{B} / W_{b}=0.0213, N_{\text {eff }}=6.05\right]$ (squares); $U / W_{B}=-0.375\left[E_{B} / W_{b}=0.0703, N_{\text {eff }}=2.38\right]$ (triangles pointing up); $U / W_{B}=-0.59\left[E_{B} / W_{b}=0.2397\right.$, $\left.N_{\text {eff }}=1.35\right]$ (triangles pointing down).

charge distribution in the trap which was calculated by the DSDMC technique (see Fig. 9).

To determine unambiguously the functional dependence $N_{e f f}=N_{e f f}\left(E_{B}\right)$ from the calculated relations $E_{B}=E_{B}(U, \lambda)$ and $N_{e f f}=N_{e f f}(U, \lambda)$ (Figs. $8 \mathrm{a}$ and 8b) we must decide which of the two parameters, $\lambda$ and $U$, is fixed and which is responsible for the variation of the physical parameters of the traps: the binding energy $E_{B}$ and the localization parameter $N_{e f f}$. A proper choice of the parameter responsible for the variation in the physical properties of the traps determines relation (17) fully and unambiguously. Since the thin film in pentacene TFTs consists solely of pentacene molecules it is natural to assume that the value of $\lambda$ is one and the same for the entire film and the spread of the physical parameters of the traps is caused by trapping potentials of different origins. The trapping potentials of different origins, in turn, can be characterized by different strengths of the attractive potentials $U$.

\section{B. Energy distribution of traps in pentacene TFTs}

To analyze the ESR spectrum of pentacene TFT we used the electron-phonon coupling constant $\lambda=0.15$, estimated from optical absorption experiments ${ }^{60}$, and the hopping amplitude $t=0.1 \mathrm{eV}$, obtained from band structure calculations 61,62 . Figure 10 shows the distribution $\mathcal{Z}\left(E_{B}\right)$ of the trapped carriers over the binding energies in TFT at the gate voltage $-200 \mathrm{~V}$. We fixed $\lambda=0.15$, considered the dependence of $E_{B}$ (bold line in Fig. $8 \mathrm{a}$ ) and $N_{\text {eff }}$ (bold line in Fig. 8 $8 \mathrm{~b}$ ) on the attractive potential $U$, and obtained $N_{\text {eff }}\left(E_{B}\right)$ (bold line in Fig. 8 c). Then, we used transformation (18).

We found that the two discrete trap levels (A and B) 


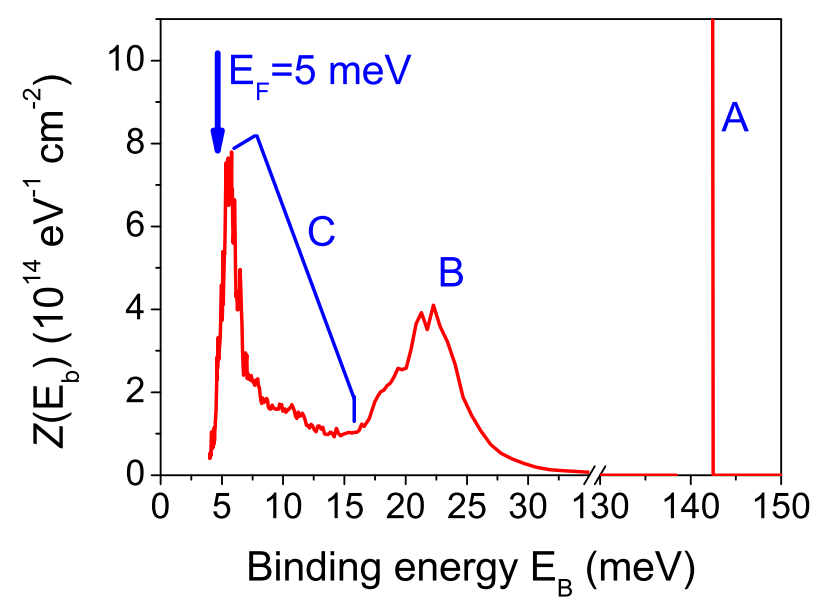

FIG. 10: (color online) Distribution of trap states in pentacene TFTs as function of trap energy $E_{B}$ for gate voltage $-200 \mathrm{~V}$.

peak at $140 \pm 50 \mathrm{meV}$ and $22 \pm 3 \mathrm{meV}$, respectively. The broad feature (C) at the gate voltage $-200 \mathrm{~V}$ is distributed between 5 and $15 \mathrm{meV}$, as presented in Fig. 10. The low-energy profile prompts the anticipation of tail states extending from just below the band edge, as has been discussed for amorphous semiconductors, while the states are partially occupied up to the Fermi level at around $E_{B}=5 \mathrm{meV}$. These results are roughly consistent with the small activation energy of about $15 \mathrm{meV}$ for the motional narrowing observed in [14], and also with the potential fluctuations by atomicforce-microscope potentiometry ${ }^{63}$. We note that the distribution $\mathcal{Z}\left(E_{B}\right)$ gives relatively correct position of the trap levels, although the absolute value of the binding energy is rather model-dependent.

\section{DISCUSSION}

Weakly-localized in-gap states are expected to play crucial roles in the intrinsic charge transport along semiconductor/gate dielectric interfaces in organic transistors. In practice, temperature-independent mobility is often observed in devices with high mobility and highlyordered molecular interfaces, which indicates that the Fermi energy is just below the band edge ${ }^{64}$.

To date, various experimental techniques have been used to investigate the interfacial trap density, such as deep-level transient spectroscopy (DLTS) ${ }^{65}$, photocurrent yield ${ }^{66}$, gate-bias stress ${ }^{67}$, and thermally-stimulated current $^{68}$ experiments. However, the measurements are based on the charge transport, and it is strongly affected by the "extrinsic" potential barriers at grain boundaries and/or channel/electrode interfaces. In striking contrast, our method has a crucial advantage in its ability to dis-

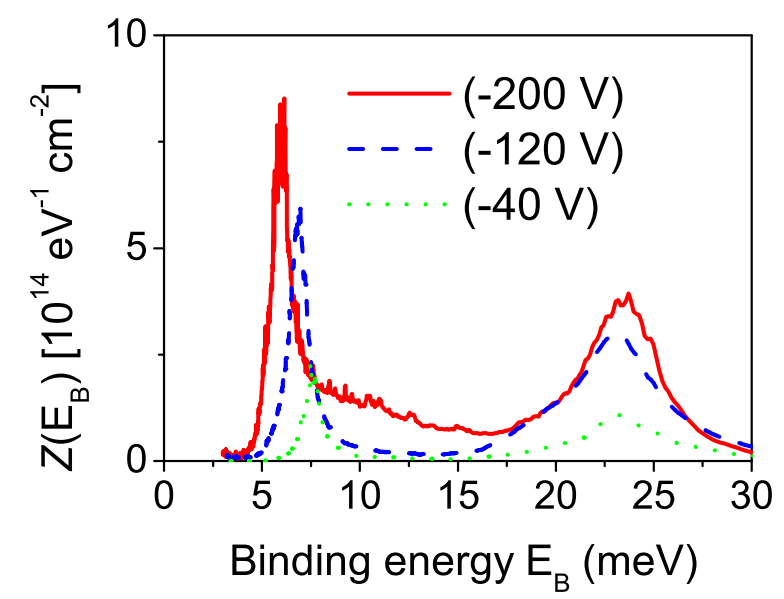

FIG. 11: (color online) Comparison of distributions of trap states in pentacene TFTs as function of trap energy $E_{B}$ for gate voltage $-200 \mathrm{~V}$ (solid line), $-120 \mathrm{~V}$ (dashed line), and -40 $\mathrm{V}$ (dotted line).

close the spatial and energy distribution of shallow traps down to a few meV, based on a unique microscopic probe using electronic spins. In addition, the g tensor can be used to identify the molecular species around the trap sites. For the three types (A, B, and C) of trap states obtained, the $\mathrm{g}$ tensor should be common, considering the highly symmetric nature of the ESR spectra. This indicates that the trap states are extended over inherent pentacene molecules of regular orientation 8.10 . Of these, the deep discrete trap level (A) might be attributed to structural defects such as grain boundaries 69 .

The shallow discrete level (B) and the broad feature (C) might be ascribed to small defects such as molecular sliding along the long axes of the molecules ${ }^{70}$, disorder induced by random dipoles in the amorphous gate dielectrics ${ }^{71,72}$, thermal off-diagonal electronic disorder ${ }^{73.74}$, and the fluctuations of the band edge ${ }^{75}$. Notice that the regular orientations of the molecules are retained in the trap states as stated above. Although these assignments are rather speculative, we believe that further microscopic investigations based on this study will provide a comprehensive view of the weakly-localized in-gap states in organic transistors.

The lack of sensitivity of the strongly localized states with small $N_{\text {eff }}$ to the gate voltage, which is obvious from the physical point of view, is an indication of the stability and reliability of the fine analysis of the ESR spectra. For shallow states with large $N_{\text {eff }}$ and small $E_{B}$, an increase in the gate voltage adds low-energy states that participate in creation of ESR signal (Fig. 11). This shift of the border where states are visible to the ESR probe indicates that these states are filled as the gate voltage increases. Hence, this behavior can be interpreted as a movement of the Fermi level which tends to zero energy 
as the bias voltage increases.

It is important to mention that the sharp peak of $D\left(N_{e f f}\right)$ at $N_{e f f}=1.54$ does not contradict the assumptions used to derive the integral equations (8) and (9). The very essence of these equations implies that the contribution from each state with a given $N_{e f f}$ is a Gaussian ESR signal. On the other hand, the signal at small $N_{\text {eff }}$ is a more complicated function with fine features (see e.g. Fig. 11 for $N_{e f f}=1$ ). The results for distribution $D\left(N_{\text {eff }}\right)$ at small $N_{\text {eff }}$ can be unreliable. However, as can be seen in Fig. 11, the ESR signal for reasonable parameters is close to Gaussian even at $N_{\text {eff }}=1.54$. Therefore, the results for the distribution of the traps $D\left(N_{e f f}\right)$ are valid even for small values of the localization parameter $N_{\text {eff }}$.

\section{CONCLUSIONS}

We have presented an unbiased analytical method for the processing of high-precision electron spin resonance (ESR) spectra, which allows us to obtain the distribution of trapped carriers over the degree of localization and the binding energy. The first step is a fine analysis of the shape of the ESR spectra by the SOM, which allows us to split the spectrum into multiple Gaussian components each of which corresponds to a different spatial extension of the trapped carriers. The second step is the transformation of the distribution over the degree of localization into a distribution over the binding energies via a system-dependent relation between the binding energies and the localization parameters of the trapped carriers. We have presented and discussed the fundamental bases of the spectral analysis, detailed algorithms for practical applications, and discussed the robustness of the analysis to experimental noise. Although the method can be applied to many systems, we consider that it is most appropriate for ESR spectra of organic TFTs for the following reasons. First, the channel materials are composed of regularly aligned organic molecules that involve multiple degrees of freedom for nuclear spin moments. This feature clearly justifies our basic assumption that a single type of trap gives the Gaussian lineshape of the ESR spectrum. Secondly, it is possible to measure the high-precision ESR spectrum because of the fairly small spin-orbit interactions of organic materials. The fieldeffect device structure also enables the control of the carrier density without introducing any randomness in the channel semiconductors.

Such a direct probe is quite unique in investigating the microscopic carrier dynamics in the organic TFTs that have attracted considerable recent attention in the field of organic electronics. We have shown that the trap states in pentacene TFTs can be classified into three major groups: deep trap states with a spatial extension of about 1.5 molecules (A), relatively shallow trap states that extend over about 5 molecules (B), and shallower trap states that extend over 6 to 20 molecules (C). These states respectively correspond to deep and shallow trap states with binding energies of $140 \mathrm{meV}(\mathrm{A})$ and $22 \mathrm{meV}$ (B), with the broad feature ranging from 5 to $15 \mathrm{meV}(\mathrm{C})$. These shallow in-gap states are crucial for understanding and improving the device performance of organic TFTs.

\section{ACKNOWLEDGMENTS}

A.S.M. was supported by RFBR 07-02-00067a. H.M. and T.H. are supported by the Japan Science and Technology Agency (JST) through the Strategic Promotion of Innovative Research and Development Program (SInnovation). They are also partly supported by the Japan Society for the Promotion of Science (JSPS) through a Grant-in-Aid for Scientific Research from the Funding Program for World-Leading Innovative R\&D on Science and Technology (FIRST program).
1 P. W. Anderson, Nobel Lecture, 1977.

2 G. Feher, Phys. Rev. 114, 1219 (1959).

3 S. A. Altshuler and B. M. Kozirev, Electron Paramagnetic Resonance (Academic Press, NY, 1964).

4 C. P. Slichter, Principles of Magnetic Resonance (Springer Series in Solid-State Sciences, 2010).

5 J. R. Bolton, J. Chem. Phys. 46, 408 (1967).

6 J. A. Weil, J. R. Bolton, J. E. Wertz, Electron Paramagnetic Resonance: Elementary Theory and Practical Applications (Wiley-Interscience, New York 1994).

7 I. C. lewis and L. S. Singer, J. Chem. Phys. 43, 2712 (1965).

8 K. Marumoto, S.-I. Kuroda, T. Takenobu, and Y. Iwasa, Phys. Rev. Lett. 97, 256603 (2006).

9 K. Marumoto, Y. Muramatsu, Y. Nagano, T. Iwata, S. Ukai, H. Ito, S. Kuroda, Y. Shimoi, and S. Abe, J. Phys. Soc. Jpn. 74, 3066 (2005).

${ }^{10}$ H. Matsui, T. Hasegawa, Y. Tokura, M. Hiraoka, and T.
Yamada, Phys. Rev. Lett. 100, 126601 (2008).

11 H. Matsui and T. Hasegawa, Mater. Res. Soc. Symp. Proc. 1154 B08-04 (2009).

12 K. Marumoto, N. Arai, H. Goto, M. Kijima, K. Murakami, Y. Tominari, J. Takeya, Y. Shimoi, H. Tanaka, S. Kuroda, T. Kaji, T. Nishikawa, T. Takenobu, and Y. Iwasa, Phys. Rev. B 83, 075302 (2011).

13 R. Kubo and K. Tomita, J. Phys. Soc. Jpn. 9, 888 (1954).

14 H. Matsui, A. S. Mishchenko, and T. Hasegawa, Phys. Rev. Lett. 104, 056602 (2010).

15 Z. Bao and J. Locklin, Organic Field-Effect Transistors (Optical Science and Engineering, CRC Press, 2007).

16 H. Klauk, Organic Electronics: Materials, Manufacturing, and Applications (Wiley-VCH, 2006).

17 G. Horowitz, M. E. Hajlaoui, and R. Hajlaoui, J. Appl. Phys. 87, 4456 (2000).

18 D. V. Lang, X. Chi, T. Siegrist, A. M. Sergent, and A. P. Ramirez, Phys. Rev. Lett. 93, 086802 (2004). 
19 O. Tal, Y. Rosenwaks, Y. Preezant, N. Tessler, C. K. Chan, and A. Kahn, Phys. Rev. Lett. bf 95, 256405 (2005).

20 M. F. Calhoun, C. Hsieh, and V. Podzorov, Phys. Rev. Lett. bf 98, 096402 (2007).

21 T. Sueyoshi, H. Fukagawa, M. Ono, S. Kera, and N. Ueno, Appl. Phys. Lett. 95, 183303 (2009).

${ }^{22}$ H. Xie, H. Alves, and A. F. Morpurgo, Phys. Rev. B 80, 245305 (2009).

${ }^{23}$ W. L. Kalb, S. Haas, C. Krellner, T. Mathis, and B. Batlogg, Phys. Rev. B 81, 155315 (2010).

24 A. S. Mishchenko, N. V. Prokof'ev, A. Sakamoto, and B. V. Svistunov Phys. Rev. B 62, 6317 (2000).

25 A. S. Mishchenko, Usp. Phys. Nauk 175, 925 (2005) [Phys. Usp. 48, 887 (2005)].

26 A. S. Mishchenko and N. Nagaosa, J. Phys. Soc. J. 75, 011003 (2006).

27 K. Vafayi and O. Gunnarsson, Phys. Rev. B 76, 035115 (2007).

28 A. S. Mishchenko, N. Nagaosa, A. Alvermann, H. Fehske, G. De Filippis, V. Cataudella, and O. P. Sushkov, Phys. Rev. B 79, 180301(R) (2009)

29 M. Berciu, A. S. Mishchenko, and N. Nagaosa, Europhys. Lett. 89, 37007 (2010)

30 G. L. Goodvin, L. Covaci, and M. Berciu, Phys. Rev. Lett. 103, 176402 (2009) .

31 V. Cataudella, G, De Filippis, F. Martone, and C. A. Perroni, Phys. Rev. B 70, 193105 (2004).

32 G, De Filippis, V. Cataudella, V. Marigliano Ramaglia, and C. A. Perroni, Phys. Rev. B 72, 014307 (2005).

33 Computational Many-Particle Physics, Lect. Notes Phys. Vol. 739, (Ed. H Fehske, R Schneider, A Weisse, (Springer, Berlin Heidelberg, 2008).

34 M. Jarrel and J. E. Gubernatis, Phys. Rep. 269, 133 (1996)

35 A. N. Tikhonov and V. Y. Arsenin, Solutions of Ill posed Problems (Winston, Washington, 1977).

36 E. Perchik (2003).

URL

http://arxih.org/abs/math-ph/0302045. Preprint.

37 A. S. Mishchenko, N. V. Prokofév, and B. V. Svistunov, Phys. Rev. B 64, 033101 (2001).

38 A. S. Mishchenko, N. V. Prokof'ev, A. Sakamoto, and B. V. Svistunov, Int. J. Mod. Phys. B 15, 3940 (2001).

39 A. S. Mishchenko, N. Nagaosa, N. V. Prokof'ev, A. Sakamoto, and B.V. Svistunov, Phys. Rev. B 66, 020301(R) (2002).

40 A. S. Mishchenko, N. Nagaosa, N. V. Prokof'ev, A. Sakamoto, and B. V. Svistunov, Phys. Rev. Lett. 91, 236401 (2003).

41 A. S. Mishchenko and N. Nagaosa, Phys. Rev. Lett. 93, 036402 (2004).

42 A. S. Mishchenko and N. Nagaosa, Phys. Rev. B 73, 092502 (2006).

43 A. S. Mishchenko and N. Nagaosa, J. Phys. Chem. Solids 67, 259 (2006).

${ }^{44}$ G. De Filippis, V. Cataudella, A. S. Mishchenko, C. A. Perroni, and J. T. Devreese, Phys. Rev. Lett. 96, 136405 (2006).

45 A. S. Mishchenko, Proceedings of the International School of Physics "Enrico Fermi", Course CLXI, 177-206 (2006).

46 A. S. Mishchenko and N. Nagaosa, Polarons in Complex Matter, Springer Series in Material Science, Springer, ed. by A. S.Alexandrov, 503-544 (2007).

47 V. Cataudella, G. De Filippis, A. S. Mishchenko, and N. Nagaosa, Phys. Rev. Lett. 99, 226402 (2007).
48 A. S. Mishchenko, in "Computational Many-Particle Physics", ed. by H. Fehske, R. Scheider and A. Weisse, Lect. Notes Phys. 739, pp. 367-395 (Springer, Berlin Heidelberg 2008).

49 A. S. Mishchenko, N. Nagaosa, Z.-X. Shen, G. De Filippis, V. Cataudella, T. P. Devereaux, C. Bernhard, K. W. Kim, and J. Zaanen, Phys. Rev. Lett. 100, 166401 (2008).

50 V. Cataudella, G. De Filippis, A. S. Mishchenko, and N. Nagaosa, J. Supercond. Nov. Magn., 22, 17 (2009).

51 A. S. Mishchenko, N. Nagaosa, A. Alvermann, H. Fehske, G. De Filippis, V. Cataudella, and O. P Sushkov, Phys. Rev. B, 79, 180301(R) (2009).

52 A. S. Mishchenko, Usp. Phys. Nauk 179, 1259 (2009) [Phys. Usp. 52, 1193 (2009)].

53 A. S. Mishchenko, Advances in Condensed Matter Physics 2010, 306106 (2010)

54 G. L. Goodvin, A. S. Mishchenko, and M. Berciu, Phys. Rev. Lett. 107, 076403 (2011).

55 A. S. Mishchenko, N. Nagaosa, K. M. Shen, Z.-X. Shen, X. J. Zhou, T. P. Devereaux, Europhys. Lett. 96, 57007 (2011).

${ }^{56}$ H. Hafermann, S. Brener, A. N. Rubtsov, M. I. Katsnelson, and A. I. Lichtenstein, J. Phys.: Condens. Matter 21, 064248 (2009).

57 H. Hafermann, M. I. Katsnelson, and A. I. Lichtenstein, Europhys. Lett., 85, 37006 (2009).

58 E. Gorelov, M. Karolak, T. O. Wehling, F. Lechermann, A. I. Lichtenstein, and E. Pavarini, Phys. Rev. Lett. 104, 226401 (2010).

59 E. Gorelov, J. Kolorenč, T. Wehling, H. Hafermann, A. B. Shick, A. N. Rubtsov, A. Landa, A. K. McMahan, V. I. Anisimov, M. I. Katsnelson, and A. I. Lichtenstein, Phys. Rev. B 82, 085117 (2010)

60 Z. Bao and J. Locklin, Organic Field-Effect Transistors, (CRC Press, Boca Raton, 2007).

61 P. Parisse, L. Ottaviano, B. Delley, and S. Picozzi, J. Phys. Condens. Matter 19, 106209 (2007).

62 A. Troisi and G. Orlandi, J. Phys. Chem. B 109, 1849 (2005).

63 N. Ohashi, H. Tomii, R. Matsubara, M. Sakai, K. Kudo, and M. Nakamura, Appl. Phys. Lett. 91, 162105 (2007).

64 T. Hasegawa and J. Takeya, Sci. Tech. Adv. Mater. 10, 024314 (2009).

65 Y. S. Yang, S. H. Kim, J.-I. Lee, H. Y. Chu, L.-Mi Do, H. Lee, J. Oh, T. Zyung, M. K. Ryu, and M. S. Jang, Appl. Phys. Lett. 80, 1595 (2002).

66 D. V. Lang, X. Chi, T. Siegrist, A. M. Sergent, and P. Ramirez, Phys. Rev. Lett. 93, 086802 (2004).

67 C. Goldman, C. Krellner, K. P. Pemstich, S. Haas, D. J. Gundlach, and B. Batlogg, J. Appl. Phys. 99, 034507 (2006).

68 T. Matsushima, M. Yahiro, and C. Adachi, Appl. Phys. Lett. 91, 103505 (2007).

69 S. Verlaak and P. Heremans, Phys. Rev. B 75, 115127 (2007).

70 J. H. Kang, D. da Silva Filho, J.-L. Bredas, and X.-Y. Zhu, Appl. Phys. Lett. 86, 152115 (2005).

71 J. Veres, S. D. Ogier, S. W. Leeming, D. C. Cupertino, and S. M. Khaffaf, Adv. Funct. Mater. 13, 199 (2003).

72 H. Houili, J. D. Picon, L. Zippiroli, and M. N. Bussac, J. Appl. Phys. 100, 023702 (2006).

73 A. Troisi and G. Orlandi, Phys. Rev. Lett. 96, 086601 (2006).

74 A. Troisi, Phys. Rev. B 82, 245202 (2010). 
75 R. Matsubara, M. Sakai, K. Kudo, N. Yoshimoto, I. Hirosawa, and M. Nakamura, Organic Electronics 12, 196

(2011). 